\title{
Growth of alumina oxide nanowires in an aluminum anodization process
}

\author{
ZHU KaiGui $^{1 *}$, CHENG JingRan ${ }^{1}$, CHANG MingChao ${ }^{1}$, WANG $\mathrm{Wu}^{1}$, WEI WenBo ${ }^{2}$ \& \\ GE GuangLu ${ }^{2}$ \\ ${ }^{1}$ Department of Physics, Beihang University, Beijing 100191, China; \\ ${ }^{2}$ Laboratory of Nanostanderization, National Center for Nanoscience and Technology, Beijing 100190, China
}

Received December 8, 2010; accepted February 23, 2011; published online May 12, 2011

\begin{abstract}
Alumina oxide nanowires were grown via a two-step anodization of aluminum foil. A growth mechanism is proposed for these nanowires which is different from that of those formed using chemical etching methods. An electric field and stress are necessary to grow these nanowires, and the nano-imprinted metal surface of the aluminum foil has an important role.
\end{abstract}

PAAO, porous alumina oxide, aluminum anodization, nanowires

Citation: Zhu K G, Cheng J R, Chang M C, et al. Growth of alumina oxide nanowires in an aluminum anodization process. Chinese Sci Bull, 2011, 56: 1947-1950, doi: 10.1007/s11434-011-4507-y

One-dimensional nanostructures have become the focus of intensive research because of their unique properties and potential applications in high density nanoscale devices including sensors [1], optoelectronics [2], and magnetic memory [3]. In the early 1990s, Brumlik et al. [4] and Martin [5] pioneered the use of hard templates in the synthesis of metal and polymer tubes, rods and wires. This was achieved through either electrochemical or electroless deposition onto a porous anodic alumina oxide (PAAO) membrane followed by dissolution of the membrane. Subsequently, this approach was widely employed to synthesize metal [3], semiconductor [1,2] and polymer [6] nanoarrays. Further research has yielded methods for producing ideally ordered-hole configurations in PAAO [7,8]. Because of this, the fabrication of carbon nanotubes with specific crosssections [9] and the spatially controlled periodic deposition of two kinds of materials [10] have been achieved. This kind of substrate can also be fabricated using polymer precursors for industrial applications [11], and used in the preparation of smart surfaces [12].

*Corresponding author (email: kgzhu@buaa.edu.cn)
Nanoscale alumina oxide structures have been obtained using electrochemical methods. Alumina oxide nanotubes with many branches were fabricated via the anodization of aluminum on a silicon substrate in dilute sulfuric acid [13]. Alumina oxide nanotubes and nanowires were produced by chemically etching PAAO membranes either in sodium hydroxide solution [14,15] or in phosphoric acid solution [15-20]. However, to the best of our knowledge, there are few reports of the formation of alumina oxide nanowires in organic acid solution. Moreover, there have been no comparisons between the growth of nanowires in an etching process and that in anodization. In this work, alumina oxide nanowires are obtained after a two-step anodization of an aluminum foil. A growth mechanism is proposed for these nanowires. This proposal should be conducive to studies on the self-ordering mechanism of cells during the anodization of porous aluminum.

\section{Experimental}

A piece of high-purity aluminum foil was flattened under an even pressure of $10 \mathrm{MPa}$. Then the foil was annealed for $5 \mathrm{~h}$ 
to release residual stress. The annealing temperature was between $400-600^{\circ} \mathrm{C}$. Prior to the electrochemical process, the aluminum foil was ultrasonically degreased with acetone and rinsed in ethanol. The prepared foil served as an anode, and two slices of graphite acted as cathodes. They were then electropolished in a mixture of $6.2 \mathrm{vol} \%$ perchloric acid, $70.1 \mathrm{vol} \%$ ethanol, 10 vol\% butyl cellosolve and 13.7 vol\% distilled water under a voltage of $30 \mathrm{~V}$ for no more than $30 \mathrm{~s}$. A specially designed polytetrafluoroethylene device that uses two hollow cylindrical components was used to secure the electropolished foil with a pair of O-rings functioning as padding. Subsequently, the device was horizontally attached to a vessel, which contained an oxalic acid solution to serve as an electrolyte. Also, it contained a slice of graphite vertically positioned face-to-face with the foil. A space, which was delimited by the cylindrical polytetrafluoroethylene shell, was left between them, and was occupied by the oxalic acid solution. The electrolyte only makes contact with one side of the foil; thus single-sided anodization is possible. Two electric wires were employed for anodization: one to bridge the foil and positive terminal of the direct current source, and the other to bridge the graphite and negative terminal.

Anodization was performed using a two-step scheme in which the aluminum foil undergoes anodization and subsequent removal of its outer oxide layer leaving a nanoimprinted metal surface. The second anodization step facilitates the self-organized growth of regular and ordered pores in the alumina oxide layer, which results in better PAAO. In our experiment, the first step lasts $4 \mathrm{~h}$, and the second one lasts $8 \mathrm{~h}$. Both steps feature an electrolyte of $0.4 \mathrm{~mol} / \mathrm{L}$ oxalic acid solution and an anodizing voltage of $40 \mathrm{~V}$. Between the two anodization steps the polytetrafluoroethylene device securing the foil was removed from the vessel and put into an $80^{\circ} \mathrm{C}$ constant temperature water bath for $1.5 \mathrm{~h}$ to remove oxide layer that formed during the first step of anodization. To efface the device we used a 1:1 vol mixture of $1.8 \mathrm{wt} \%$ chromic acid solution and $6 \mathrm{wt} \%$ phosphoric acid solution. The voltage was gradually reduced to $0 \mathrm{~V}$ in the last stage of the second anodization step to extend the barrier alumina oxide layer beneath the porous one [21]. A $1: 1$ vol mixture of $5 \mathrm{wt} \% \mathrm{CuCl}_{2}$ solution and $20 \mathrm{wt} \% \mathrm{HCl}$ solution was used to expunge the unanodized side of the aluminum. Finally, the exposed barrier layer, which was previously sandwiched between the porous layer and the metal aluminum one, was corroded using $5 \mathrm{wt} \%$ phosphoric acid in a $60^{\circ} \mathrm{C}$ constant temperature water bath for $1.5 \mathrm{~h}$.

\section{Results and discussions}

A scanning electron microscopy (SEM) study of the samples after the second oxidization step showed that nanowires reside on top of the porous alumina oxide layer in the peripheral zones of the sample. Their roots extend into the porous layer and morph into nexuses, which each bridge three adjacent pore walls. This is shown in Figure 1, whose inset shows a clear line between the porous and nanowire abundant areas. Figure 2 shows the nanowires at a lower magnification, in which one can see that they lie in a stretching and cascading pattern. The upper right inset of Figure 2 displays the nanowires in a magnified view. These nanowires are about tens of nanometers in diameter and tens of microns in length, which yields a length/diameter ratio above one hundred. An energy-dispersive X-ray spectroscopy analysis in the upper left inset of Figure 2 shows that these nanowires consist of $\mathrm{Al}$ and $\mathrm{O}$ in an atomic ratio of 27.63:68.15. There are some impurity elements mixed into the sample, such as Pt, which was sputtered on the sample to improve its conductivity.

Previous studies confirm the formation of alumina oxide nanowires via the immersion of PAAO into various kinds of acid or alkaline solutions $[14,16]$. Nanowires, which grew as a consequence of the treatment using $10 \mathrm{wt} \%$ sulfuric

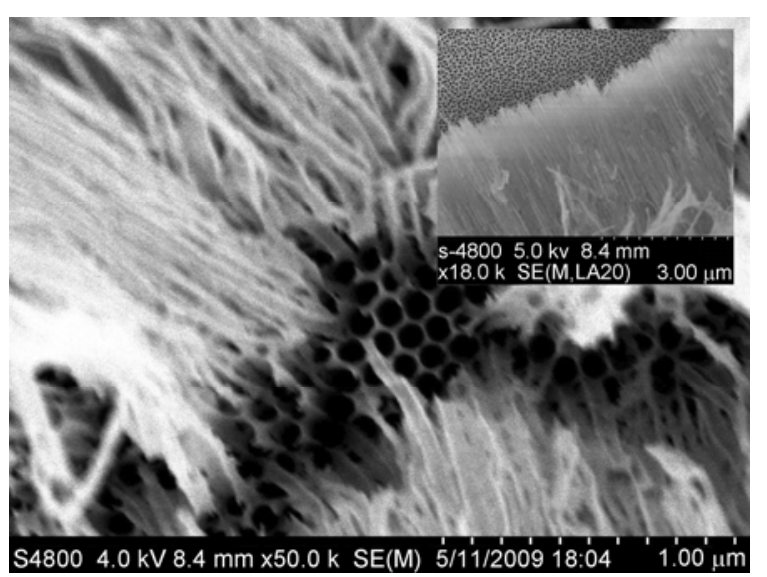

Figure 1 Nanowires with their roots extending into the porous layer on the porous anodic alumina oxide, which was anodized in an oxalic acid solution. Inset: Nanowires lying prostrate on the porous anodic alumina oxide.

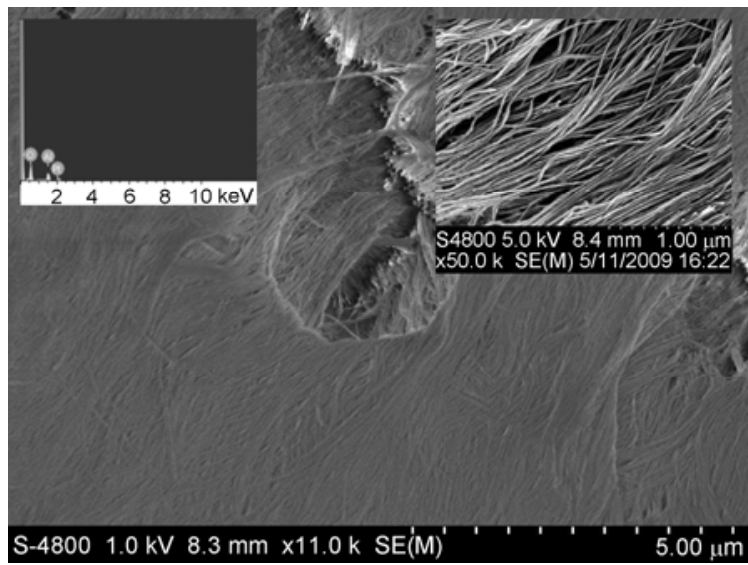

Figure 2 Nanowires in a stretching and cascading pattern obtained after anodization of the porous anodic alumina oxide in oxalic acid solution. Upper right inset: The nanowires in a magnified view. Upper left inset: Energy-dispersive X-ray spectroscopy analysis of the nanowires. 
acid solution, were concentrated on top of the undissolved porous alumina oxide layers. This shaped a plateau through the fissures in which a portion of the nanowires are exposed. This is shown in Figure 3. A magnified image of the exposed nanowires is shown in the inset of Figure 3. From this image, it can be seen that the fiber-like matter aggregated forming a spongy plateau.

Two intermediate samples were examined using SEM: one after the first oxidization step, and the other after the removal of the first oxide layer. These results show that there are no nanowires on the first sample. Also, a small amount of nanowires formed in the peripheral zones of the second sample. These nanowires are similar in irregularity and arrangement to those formed by the immersion of PAAO in sulfuric acid solution. These facts are indicative of the significance of the electric field and nano-imprinted metal surface of the aluminum foil, which resulted from the first oxidization step and the subsequent removal of the outer oxide layer formed during the second oxidization step. However, these two conditions are insufficient to ensure the growth of the nanowires, which also requires the stress in aluminum foil created by the pair of O-rings.

At the beginning of the second anodization step, the stress gives rise to many crevasses along certain depressions in the surface of the aluminum foil. From these crevasses, mounds of alumina oxide material emerge. In trough sites, which are each lined by two neighboring mounds, dissolution is facilitated because of the increased intensity of the electric field, but dissolution of the mounds is comparatively moderate. Therefore, the mounds are further elevated compared with the trough sites. This makes the long nanowires possible. Pores grow everywhere on the metal surface, which is typical of anodizing processes. This works in tandem with the dissolution in the trough sites. Also, the mild dissolution of the mounds takes place chiefly in a counter-radial pattern because of the comparatively strong activity of the oxalic acid solution at the brink of the mounds. Hence, a mound with pores inside can transform into a bunch of nanowires.

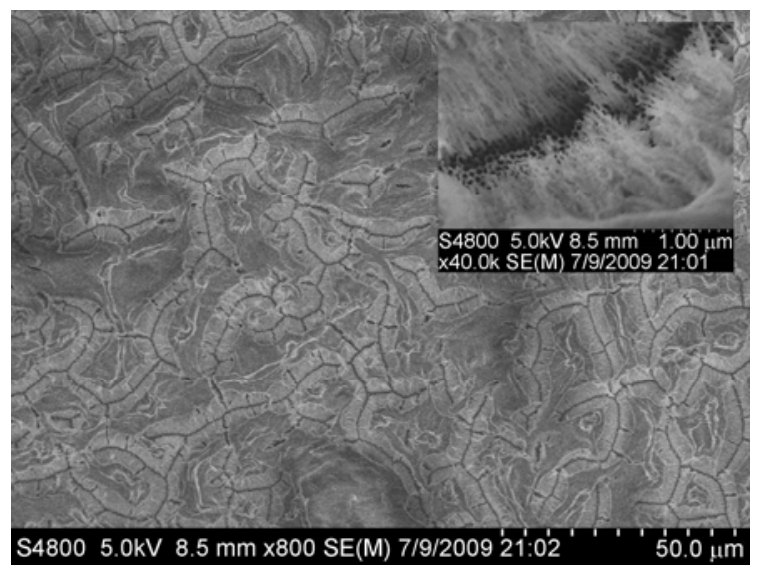

Figure 3 Overview of porous anodic alumina oxide etched using a $10 \mathrm{wt} \%$ sulfuric acid solution. Inset: Magnified image of the spongy etched porous anodic alumina oxide.
The pore walls flake and thicker nexuses, which each bridge three adjacent pore walls, remain. This is unlike the central zones where dissolution proceeds in a uniform manner rather than forming pores. The remains of the mounds can be seen as clusters of nanowires in Figure 4, in which the nanowires are not long enough to mask the cluster profiles. The nanowires in the inset of Figure 4 are in an embryotic state, in which trough sites are still visible. Stress exists mainly in the peripheral zones. Therefore, it is reasonable to expect that nanowires would only grow in these zones. This flaking dissolution may result in nanowires of diameters independent of the pore size of the PAAO and the growth of nanotubes [14] of the same inner diameter as the pores. It is possible that these nanotubes form given the variation in the pore size and the distance between pores.

The electric field is not present in the case [16] of etching PAAO using a phosphoric acid solution, and a nonuniform electric field develops in the peripheral zones of the sample in this paper. This indicates that pores and nanowires can form when corrosive solutions make contact with aluminum or alumina oxide. However, the presence of an adequate uniform electric field can make the formation of pores favorable. The reason why we found few nanowires in the central zones of alumina oxide material is that they are preferentially dissolved there. An electric field may be incentive for relatively uniform dissolution of alumina oxide material, but cracks on the surface of PAAO disrupt this process. In particular, this can occur when the electric field is not uniform. For example, stress in the metal in this study can lead to the growth of nanowires in peripheral zones.

The formation of the nanowires during the removal of the first oxide layer is primarily the result of the etching of a comparatively thin layer of nonuniform PAAO on an aluminum substrate in the presence of stress. This explains the small differences between this kind of nanowire and the kind formed by treating PAAO with acid or alkaline solutions. These nanowires have no effect on the nanowires formed after the second step of oxidization because an

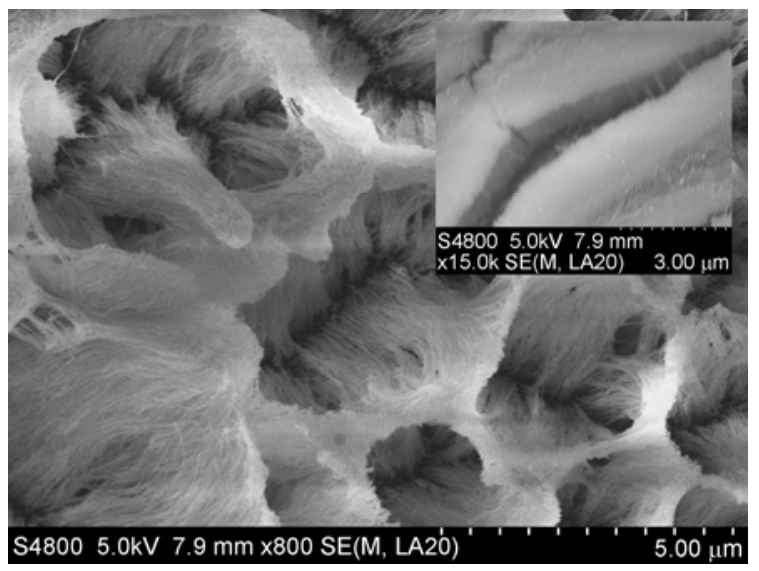

Figure 4 Clusters of nanowires in the early stages of growth on porous anodic alumina oxide anodized in oxalic acid solution. Inset: Nanowires in an even earlier stage. 
identical result could be observed in an experiment performed on a sample devoid of nanowires prior to the second oxidization step.

\section{Conclusions}

The growth of alumina oxide nanowires was observed in a two-step anodizing process. SEM was employed to characterize these nanowires. An electric field develops in the delimited cylindrical space between the graphite slice and the aluminum foil. This field works in tandem with the stress within the foil created by the O-ring device to guarantee the stable growth of these nanowires.

This work was supported by the China Beijing Outstanding Talent Funding (321530), the National Basic Research Program of China (2009GB106003) and the Student Research Training Program of Beihang University of China.

1 Peng T, Yang H, Dai K, et al. Fabrication and characterization of $\mathrm{CdS}$ nanotube arrays in porous anodic aluminum oxide templates. Chem Phys Lett, 2003, 379: 432-436

2 Miao Z, Xu D, Ouyang J, et al. Electrochemically induced sol-gel preparation of single-crystalline $\mathrm{TiO}_{2}$ nanowires. Nano Lett, 2002, 2: 717-720

3 Qin J, Nogues J, Mikhaylova M, et al. Differences in the magnetic properties of $\mathrm{Co}, \mathrm{Fe}$, and $\mathrm{Ni} 250-300 \mathrm{~nm}$ wide nanowires electrodeposited in amorphous anodized alumina templates. Chem Mater, 2005, 17: 1829-1834

4 Brumlik C J, Martin C R. Template synthesis of metal microtubules. J Am Chem Soc, 1991, 113: 3174-3175

5 Martin C R. Membrane-based synthesis of nanomaterials. Chem Mater, 1996, 8: 1739-1746

6 Wang C, Wang Z, Li M, et al. Well-aligned polyaniline nano-fibril array membrane and its field emission property. Chem Phys Lett, 2001, 341: 431-434
7 Masuda H, Yamada H, Satoh M, et al. Highly ordered nanochannel-array architecture in anodic alumina. Appl Phys Lett, 1997, 71: 2770-2772

8 Asoh H, Nishio K, Nakao M, et al. Conditions for fabrication of ideally ordered anodic porous alumina using pretextured Al. J Electrochem Soc, 2001, 148: B152-B156

9 Yanagishita T, Sasaki M, Nishio K, et al. Carbon nanotubes with a triangular cross-section, fabricated using anodic porous alumina as the template. Adv Mater, 2004, 16: 429-432

10 Masuda H, Abe A, Nakao M, et al. Ordered mosaic nanocomposites in anodic porous alumina. Adv Mater, 2003, 15: 161-164

11 Liu X, Wu W, Wang X, et al. A replication strategy for complex micro/nanostructures with superhydrophobicity and superoleophobicity and high contrast adhesion. Soft Matter, 2009, 5: 3097-3105

12 Liu X, Ye Q, Yu B, et al. Switching water droplet adhesion using responsive polymer brushes. Langmuir, 2010, 26: 12377-12382

13 Zou J, Pu L, Bao X, et al. Branchy alumina nanotubes. Appl Phys Lett, 2002, 80: 1079-1081

14 Xiao Z, Han C Y, Welp U, et al. Fabrication of alumina nanotubes and nanowires by etching porous alumina membranes. Nano Lett, 2002, 2: 1293-1297

15 Zhang L, Yao S, Zhang W, et al. Preparation and formation mechanism of alumina nanowires (in Chinese). Acta Phys Chim Sin, 2005, 11: $1254-1258$

16 Mei Y, Siu G, Fu R K Y, et al. Formation mechanism of alumina nanotubes and nanowires from highly ordered porous anodic alumina template. J Appl Phys, 2005, 97: 034305

17 Liu W, Du S, Tan J, et al. Effects of etching condition on preparation of alumina nanowires (in Chinese). Mater Sci Eng Powder Metall, 2009, 14: 100-104

18 Chen L, Zheng R, Cheng G, et al. Preparation of alumina nanowires by chemical method (in Chinese). Nonferr Metal Soc, 2005, 15: 185-188

19 Xu J, Liu W, Cheng G, et al. Synthesis and optical properties of large-scale aligned alumina nanowire arrays. Mod Phys Lett B, 2010, 24: $151-160$

20 Wu W, Wang X, Wang D, et al. Alumina nanowire forests via unconventional anodization and super-repellency plus low adhesion to diverse liquids. Chem Commun, 2009, 9: 1043-1045

21 Jessensky O, Muller F, Gosele U. Self-organized formation of hexagonal pore arrays in anodic alumina. Appl Phys Lett, 1998, 72: 1173-1175

Open Access This article is distributed under the terms of the Creative Commons Attribution License which permits any use, distribution, and reproduction in any medium, provided the original author(s) and source are credited. 